\title{
Localization of Plasmon Resonance Using Momentum-Resolved EELS
}

\author{
Eric R. Hoglund ${ }^{1}$ and James M. Howe ${ }^{1}$
}

1. Dept. of Materials Science \& Engineering, University of Virginia, Charlottesville, VA, USA.

Electron energy loss spectroscopy (EELS) in a scanning transition electron microscope (STEM) is an indispensable tool for evaluating the electronic state of materials with high spatial resolution. When considering spatially resolved EELS one typically turns to a core-loss edge that has a large energy loss and small cross section. Low-energy excitations are typically more delocalized; for instance, a plasmon in Al typically has a delocalization of about $3 \mathrm{~nm}$. This primarily results from the dipole nature of plasmon excitation as an electron passes an "electron cloud" and causes a many-body resonance. Such resonance is typically derived from dielectric theory treating the electrons as non-interacting, although each electron can exchange momenta with other electrons. Other formulations such as the Lindhard model account for such interactions and result in a plasmon resonance that is momentum $(\boldsymbol{q})$ dependent [1]. Despite their relatively large delocalization, plasmons have been employed to determine important material parameters such as grain boundary energies [2] and mechanical properties [3], as well as characterizing beam sensitive materials [4] and distinguishing between allotropes of carbon [5]. If the localization and spatial sensitivity of plasmons were improved, their utility in materials characterization would further increase. Such hurdles have been overcome in vibrational EELS of polar materials using momentum-resolved EELS ( $q$-EELS), where there is a transition from dipolar excitation to impact scattering when off-axis geometries are employed [6]. To this end, the plasmon resonance across a vacuum/Al interface was examined using $\boldsymbol{q}$ EELS to take advantage of the dispersive nature of plasmons and localize its response.

The $\boldsymbol{q}$-EELS experiments were performed using a FEI Titan 80-300 S/TEM operating at $300 \mathrm{kV}$ with a Tridiem Model 863P Gatan imaging filter. Line scans were performed across a vacuum/Al interface with a convergence angle of $2.1 \mathrm{mrad}\left(1.09 \mathrm{~nm}^{-1}\right)$, thereby allowing for sampling of $\boldsymbol{q}$-space between diffraction discs. The area of interest between diffraction discs was aligned with the optic axis using the diffraction shift coils so that it passed through a $2 \mathrm{~mm}$ entrance aperture (EA) while maintaining a constant incidenceangle to the sample. The sample was oriented to a $<110>$ zone-axis so the 000 forward-scattered beam, 002 Brillouin zone (BZ), $\overline{1} 11 \mathrm{BZ}$, and $1 \overline{1} 1 \mathrm{BZ}$ were accessible. A line scan was acquired for each of the four regions at a camera length of $160 \mathrm{~mm}$, which resulted in the $2 \mathrm{~mm}$ EA accepting $2.0 \mathrm{mrad}\left(1.00 \mathrm{~nm}^{-}\right.$ $\left.{ }^{1}\right)$. Each line scan simultaneously recorded the annular dark-field (ADF) signal and energy loss spectra (ELS). The resulting zero loss peak (ZLP) of the ELS were aligned using HyperSpy software [7]. The ranges of $-5-5 \mathrm{eV}, 5-10 \mathrm{eV}$, and $10-20 \mathrm{eV}$ were integrated to provide the relative intensity variation of the ZLP, surface plasmon (SP), and volume plasmon (VP) are shown in Fig. 1.

The first common signal among all acquisitions was the ADF signal. The non-abrupt change in signal indicated thickness variations or probe broadening that results from the low convergence angles. Fitting the ADF signal with an error-function quantified the probe full-width at half-maximum as $5.2 \pm 0.2 \mathrm{~nm}$, which was corroborated by directly imaging the probe. The 000 beam displayed typical behavior of a delocalized SP and VP, with the SP extending much further into the vacuum. For each of the BZ acquisitions it was found that all signals became more localized and in fact, exhibited the same impact parameter as the probe radius. This is indicative of increased localization of all signals. The ZLP signal is a result of quasi-elastic (QE) interaction in the sample, which due to its low energy, may plural scatter with a plasmon resulting in a non-dispersive $15 \mathrm{eV}$ peak observable at non-zero $\boldsymbol{q}$. In contrast, the $\mathrm{QE}$ 
interaction diverges from the VP signal for all three BZ acquisitions, indicating that the integrated VP intensity does not result from a plural-scattering peak. Another feature to note is that the VP displays anisotropy at $\boldsymbol{q} \neq 0$. For instance, the $\overline{1} 11 \mathrm{BZ}$ VP signal parallels the ADF signal, while the $1 \overline{1} 1$ and 002 BZ VP's are peaked precisely at the interface. Based on these results, it is concluded that $\boldsymbol{q}$-EELS enables observation of the anisotropic behavior of the electron gas across a vacuum/Al interface with a plasmon signal localized to the diameter of the $5 \mathrm{~nm}$ probe, and these behaviors need to be explained by different scattering mechanics than the typical delocalized dipolar interaction.

\section{References:}

[1] FJ García de Abajo, Rev. Mod. Phys. 82 (1) (2010), p. 209.

[2] P Nandi et al., Microsc. Microanal. 23 (S1) (2017), p. 414.

[3] VP Oleshko et al., Microsc. Microanal. 8 (4) (2002), p. 350.

[4] M Boniface et al., Nano Lett. 16 (12) (2016), p. 7381.

[5] RF Egerton and MJ Whelan, J. Electron Spectrosc. Relat. Phenom. 3 (3) (1974), p. 232.

[6] FS Hage et al., Phys. Rev. Lett. 122 (1) (2019).

[7] F de la Peña et al., hyperspy/hyperspy v1.4.1. (2018).
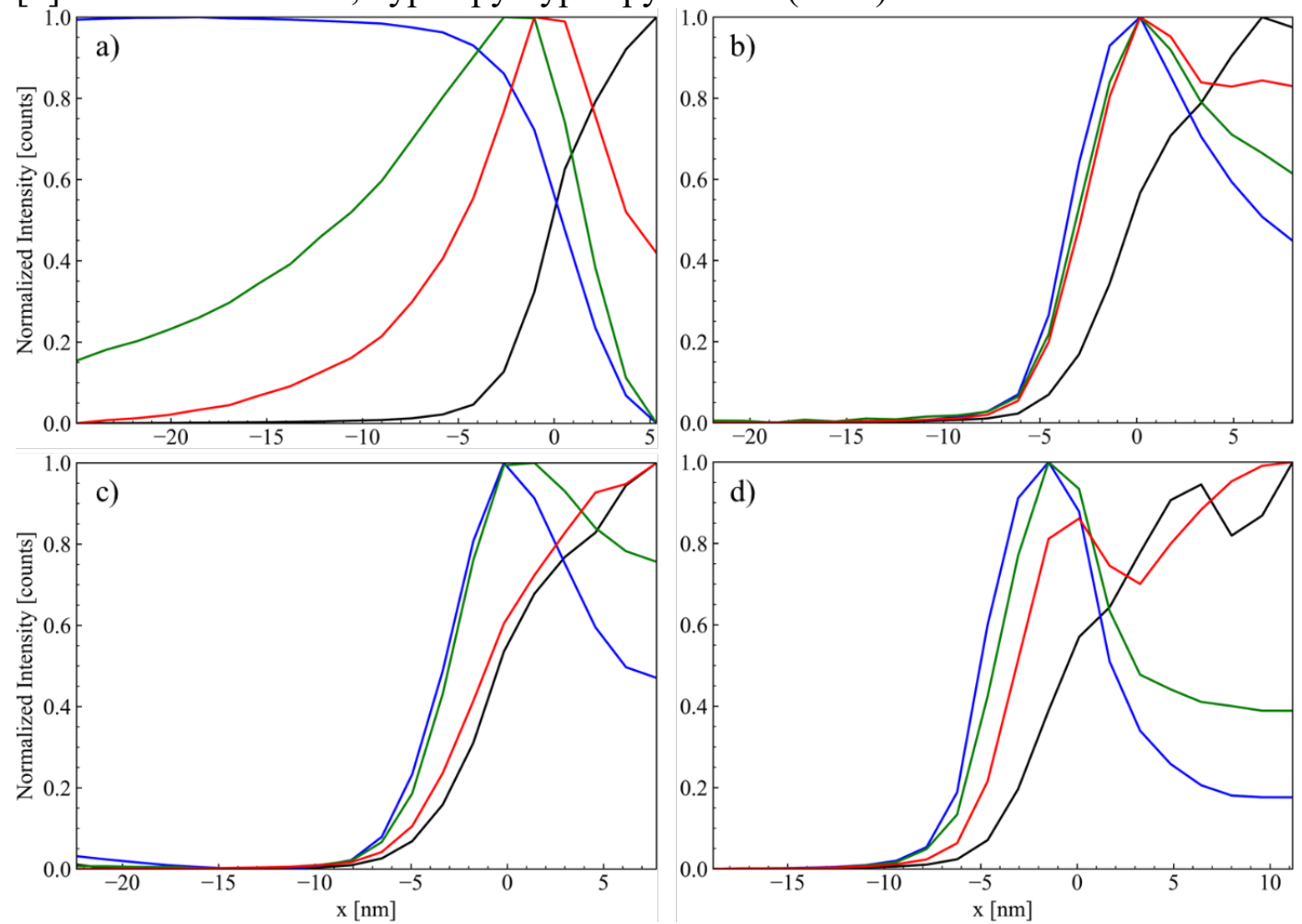

Figure 1. Cumulative intensity of the ZLP (blue), surface plasmon (green), and volume plasmon (red) for the: a) 000 beam, b) $002 \mathrm{BZ}$, c) $\overline{1} 11 \mathrm{BZ}$, and d) $1 \overline{1} 1 \mathrm{BZ}$ aligned with the optic-axis. The ADF (black) is also shown for reference. 